\title{
SINGULAR TRAJECTORIES OF CONTROL-AFFINE SYSTEMS
}

\author{
YACINE CHITOUR ${ }^{\dagger}$, FRÉDÉRIC JEAN J AND EMMANUEL TRÉLAT $^{\S}$
}

\begin{abstract}
When applying methods of optimal control to motion planning or stabilization problems, some theoretical or numerical difficulties may arise, due to the presence of specific trajectories, namely, singular minimizing trajectories of the underlying optimal control problem. In this article, we provide characterizations for singular trajectories of control-affine systems. We prove that, under generic assumptions, such trajectories share nice properties, related to computational aspects; more precisely, we show that, for a generic system - with respect to the Whitney topology -, all nontrivial singular trajectories are of minimal order and of corank one. These results, established both for driftless and for control-affine systems, extend results of 13, 14. As a consequence, for generic systems having more than two vector fields, and for a fixed cost, there do not exist minimizing singular trajectories. We also prove that, given a control system satisfying the LARC, singular trajectories are strictly abnormal, generically with respect to the cost. We then show how these results can be used to derive regularity results for the value function and in the theory of Hamilton-Jacobi equations, which in turn have applications for stabilization and motion planning, both from the theoretical and implementation issues.
\end{abstract}

1. Introduction. When addressing standard issues of control theory such as motion planning and stabilization, one may adopt an approach based on optimal control, e.g., Hamilton-Jacobi type methods and shooting algorithms. One is then immediately facing intrinsic difficulties due to the possible presence of singular trajectories. It is therefore important to characterize these trajectories, by studying in particular their existence, optimality status, and the related computational aspects. In this paper, we provide answers to the aforementioned questions for control-affine systems, under generic assumptions, and then investigate consequences in optimal control and its applications.

Let $M$ be a smooth (i.e. $C^{\infty}$ ) manifold of dimension $n$. Consider the control-affine system

$$
\dot{x}=f_{0}(x)+\sum_{i=1}^{m} u_{i} f_{i}(x),
$$

where $x \in M, m$ is a positive integer, $\left(f_{0}, \ldots, f_{m}\right)$ is a $(m+1)$-tuple of smooth vector fields on $M$, and the control $u=\left(u_{1}, \ldots, u_{m}\right)$ takes values in an open subset $\Omega$ of $\mathbb{R}^{m}$. For $x_{0} \in M$ and $T>0$, a control $u \in L^{\infty}([0, T], \Omega)$ is said to be admissible if the trajectory $x\left(\cdot, x_{0}, u\right)$ of $(\Sigma)$ associated to $u$ and starting at $x_{0}$ is well defined on $[0, T]$. On the set $\mathcal{U}_{x_{0}, T}$ of admissible controls, define the end-point mapping by

$$
E_{x_{0}, T}(u):=x\left(T, x_{0}, u\right) .
$$

It is classical that $\mathcal{U}_{x_{0}, T}$ is an open subset of $L^{\infty}([0, T], \Omega)$ and that $E_{x_{0}, T}: \mathcal{U}_{x_{0}, T} \rightarrow M$ is a smooth map.

DeFINITION 1.1. A control $u \in \mathcal{U}_{x_{0}, T}$ is said to be singular if $u$ is a critical point of the end-point mapping $E_{x_{0}, T}$, i.e. its differential at $u, D E_{x_{0}, T}(u)$, is not surjective. A trajectory $x\left(\cdot, x_{0}, u\right)$ is said to be singular if $u$ is singular and of corank one if the codimension in the tangent space of the range of $E_{x_{0}, T}(u)$ is equal to one.

\footnotetext{
${ }^{\dagger}$ Labo. des Signaux et Systèmes, Université Paris-Sud, CNRS, Supélec, 91192 Gif-sur-Yvette cedex, France (Yacine.Chitour@lss.supelec.fr).

${ }^{\ddagger}$ ENSTA, UMA, 32 bld Victor, 75739 Paris, France (Frederic.Jean@ensta.fr).

§Université Paris-Sud, Math., UMR 8628, Bat. 425, 91405 Orsay cedex, France (Emmanuel.Trelat@math.u-psud.fr).
} 
In other words, a control $u \in \mathcal{U}_{x_{0}, T}$ is singular if the linearized system along the trajectory $x\left(\cdot, x_{0}, u\right)$ is not controllable on $[0, T]$. Singular trajectories appear as singularities in the set of trajectories of $(\Sigma)$ joining two given points, and hence, they play a crucial role in variational problems associated to $(\Sigma)$ and in optimal control, as described next.

Let $x_{0}$ and $x_{1}$ be two points of $M$, and $T>0$. Consider the following optimal control problem: among all the trajectories of $(\Sigma)$ steering $x_{0}$ to $x_{1}$, determine a trajectory minimizing the cost

$$
C_{U, \alpha, g}(T, u)=\int_{0}^{T}\left(\frac{1}{2} u(t)^{T} U(x(t)) u(t)+\alpha(x(t))^{T} u(t)+g(t, x(t))\right) d t,
$$

where $\alpha=\left(\alpha_{1}, \ldots, \alpha_{m}\right) \in C^{\infty}\left(M, \mathbb{R}^{m}\right), g \in C^{\infty}(\mathbb{R} \times M)$, and $U$ takes values in the set of symmetric positive definite $m \times m$ matrices.

According to the Pontryagin Maximum Principle (see [21]), for every optimal trajectory $x(\cdot):=x\left(\cdot, x_{0}, u\right)$, there exists a nonzero pair $\left(\lambda(\cdot), \lambda^{0}\right)$, where $\lambda^{0}$ is a nonpositive real number and $\lambda(\cdot)$ is an absolutely continuous function on $[0, T]$ (called adjoint vector) with $\lambda(t) \in T_{x(t)}^{*} M$, such that, almost everywhere on $[0, T]$,

$$
\begin{aligned}
& \dot{x}(t)=\frac{\partial H}{\partial \lambda}\left(t, x(t), \lambda(t), \lambda^{0}, u(t)\right), \\
& \dot{\lambda}(t)=-\frac{\partial H}{\partial x}\left(t, x(t), \lambda(t), \lambda^{0}, u(t)\right), \\
& \frac{\partial H}{\partial u}\left(t, x(t), \lambda(t), \lambda^{0}, u(t)\right)=0,
\end{aligned}
$$

where

$$
H\left(t, x, \lambda, \lambda^{0}, u\right):=\sum_{i=1}^{m} u_{i}\left\langle\lambda, f_{i}(x)\right\rangle+\lambda^{0}\left(\frac{1}{2} u^{T} U(x) u+\alpha(x)^{T} u+g(t, x)\right)
$$

is the Hamiltonian of the system. An extremal is a 4-tuple $\left(x(\cdot), \lambda(\cdot), \lambda^{0}, u(\cdot)\right)$ solution of the system of equations (1.2). The extremal is said to be normal if $\lambda^{0} \neq 0$ and abnormal if $\lambda^{0}=0$.

The relevance of singular trajectories in optimal control lies in the fact that they are exactly the projections of abnormal extremals. Note that a singular trajectory may be the projection of several abnormal extremals, and also of a normal extremal. A singular trajectory is said to be strictly abnormal if it is not the projection of a normal extremal. Notice that a singular trajectory is of corank one if and only if it admits a unique (up to scalar normalization) abnormal extremal lift; it is strictly abnormal and of corank one if and only if it admits a unique extremal lift which is abnormal.

For a normal extremal, it is standard to adopt the normalization $\lambda^{0}=-1$, and to derive the control $u$ as the feedback function of $(x, \lambda)$

$$
u(t)=\left(\begin{array}{c}
u_{1}(t) \\
\vdots \\
u_{m}(t)
\end{array}\right)=U(x(t))^{-1}\left(\begin{array}{c}
h_{1}(t)-\alpha_{1}(x(t)) \\
\vdots \\
h_{m}(t)-\alpha_{m}(x(t))
\end{array}\right)
$$

for every $t \in[0, T]$, where $h_{i}(t):=\left\langle\lambda(t), f_{i}(x(t))\right\rangle$, for $i=1, \ldots, m$. In particular, normal extremals are smooth on $[0, T]$. 
For abnormal extremals, the situation is much more involved, since equations (1.2) do not provide directly an expression for abnormal controls. Abnormal extremals may be nonsmooth, and it is not always possible to determine an explicit expression for singular controls. Indeed, it follows from (1.2) that

$$
h_{i}(\cdot) \equiv 0 \text { on }[0, T], i=1, \ldots, m,
$$

along every abnormal extremal. At that point, in order to compute the singular control, one usually differentiates iteratively (1.4) with respect to $t$, until the control appears explicitly (in an affine way). To recover the control, an invertibility property is then required, which may not hold in general.

In this paper, we prove that, in a generic context, such an invertibility property is obtained with a minimal number of differentiations (cf. Theorem 2.6). This is the concept of minimal order, defined in Definition 2.5. Here, genericity means that the $(m+1)$-tuple $\left(f_{0}, \ldots, f_{m}\right)$ belongs to an open and dense subset of the set of vector fields equipped with the Whitney topology. The corank one property is also proved to hold generically. We obtain similar results in the driftless case (cf. Theorem 2.16).

In a preliminary step for deriving the above results, we establish a theorem of independent interest, asserting that any trajectory of a generic control-affine system satisfies $\dot{x}=0$ almost everywhere on the set where the vector fields are linearly dependent (cf. Theorem 2.1).

When considering optimal control problems, singular minimizing trajectories may exist, and play a major role, since they are not dependent on the specific minimization problem. The issue of such minimizing trajectories was already well known in the classical theory of calculus of variations (see for instance [9, 32]) and proved to be a major focus, during the forties, when the whole domain eventually developed into optimal control theory (cf $[10]$ ). The optimality status of singular trajectories was chiefly investigated in [11, 30] in relation to control-affine systems with $m=1$, in [1, 18, 19, 30] regarding driftless systems with $m=2$ and in [2, 27 for general nonlinear control systems.

In this paper, we prove that, for generic systems with $m \geqslant 2$ (and $m \geqslant 3$ in the driftless case), and for a fixed cost $C_{U, \alpha, g}$, there does not exist minimizing singular trajectories (cf. Corollaries 2.9 and 2.19). We also prove that, given a fixed system $(\Sigma)$, singular trajectories are strictly abnormal, generically with respect to the cost (1.1) (cf. Propositions 2.12 and 2.21). We then show how the abovementioned results can be used to derive regularity results for the value function and in the theory of Hamilton-Jacobi equations, which in turn have applications for stabilization and motion planning.

This paper is organized as follows. Section 2 is devoted to the statement of the main results, firstly in the control-affine case, and secondly in the driftless case. The consequences are detailed in Section 3 , and proofs are provided in Section 4.

2. Statement of the main results. Let $M$ be a smooth, $n$-dimensional manifold. Throughout the paper, $\operatorname{VF}(M)$ denotes the set of smooth vector fields on $M$, endowed with the $C^{\infty}$ Whitney topology.

2.1. Trajectories of control-affine systems. Let $T$ be a positive real number. Consider the control-affine system

$$
\dot{x}(t)=f_{0}(x(t))+\sum_{i=1}^{m} u_{i}(t) f_{i}(x(t)),
$$


where $\left(f_{0}, \ldots, f_{m}\right)$ is an $(m+1)$-tuple of smooth vector fields on $M$, and the set of admissible controls $u=\left(u_{1}, \ldots, u_{m}\right)$ is an open subset of $L^{\infty}([0, T], \Omega)$.

For every trajectory $x(\cdot):=x\left(\cdot, x_{0}, u\right)$ of (2.1), define $I_{\mathrm{dep}}(x(\cdot))$ as the closed subset of $[0, T]$

$$
I_{\mathrm{dep}}(x(\cdot)):=\left\{t \in[0, T] \mid \operatorname{rank}\left\{f_{0}(x(t)), \ldots, f_{m}(x(t))\right\}<m+1\right\} .
$$

Note that, on the open subset of $\mathbb{R}^{n}$ where $\operatorname{rank}\left\{f_{0}, \ldots, f_{m}\right\}=m+1$, there is a one-to-one correspondence between trajectories and controls. In contrast, on $I_{\text {dep }}(x(\cdot))$, there is no uniqueness of the control associated to $x(\cdot)$; in particular, $x(\cdot)$ may be associated to both singular and nonsingular controls. This fact emphasizes the following result, which describes, in a generic context, trajectories on the subset of $\mathbb{R}^{n}$ where $\operatorname{rank}\left\{f_{0}, \ldots, f_{m}\right\}<m+1$.

THEOREM 2.1. Let $m<n$ be a nonnegative integer. There exists an open and dense subset $O_{m+1}$ of $\operatorname{VF}(M)^{m+1}$ so that, if the $(m+1)$-tuple $\left(f_{0}, \ldots, f_{m}\right)$ belongs to $O_{m+1}$, then every trajectory $x(\cdot)$ of the associated control-affine system $\dot{x}=f_{0}(x)+$ $\sum_{i=1}^{m} u_{i} f_{i}(x)$ verifies

$$
\dot{x}(t)=0, \text { for a.e. } t \in I_{\mathrm{dep}}(x(\cdot)) .
$$

In addition, for every integer $N$, the set $O_{m+1}$ can be chosen so that its complement has codimension greater than $N$.

REMARK 2.2. At the light of the previous result, one can choose the admissible control $u$ on $I_{\text {dep }}(x(\cdot))$ such that, for every $t \in I_{\text {dep }}(x(\cdot)), u(t)$ consists of any $m$-tuple $\left(\alpha_{1}, \ldots, \alpha_{m}\right)$ so that

$$
f_{0}(x(t))+\sum_{i=1}^{m} \alpha_{i} f_{i}(x(t))=0 .
$$

In particular, on any subinterval of $I_{\text {dep }}(x(\cdot))$, the trajectory $x(\cdot)$ is constant, and the control can be chosen constant as well.

REMARK 2.3. A trajectory $x(\cdot)$ is said to be trivial if it reduces to a point; otherwise it is said to be nontrivial. It is clear that, if $I_{\text {dep }}(x(\cdot)) \neq[0, T]$, then $\dot{x}(t) \neq 0$ for $t \notin I_{\text {dep }}(x(\cdot))$ and $x(\cdot)$ is nontrivial.

Let $x(\cdot)$ be a trajectory of a control-affine system associated to an $(m+1)$-tuple of $O_{m+1}$. As a consequence of Theorem 2.1, $x(\cdot)$ is trivial if and only if $I_{\text {dep }}(x(\cdot))=$ $[0, T]$.

2.2. Singular trajectories. Recall that a singular trajectory $x(\cdot)$ is the projection of an abnormal extremal $(x(\cdot), \lambda(\cdot))$. For $t \in[0, T]$ and $i, j \in\{0, \ldots, m\}$, we define

$$
h_{i}(t):=\left\langle\lambda(t), f_{i}(x(t)\rangle, \quad h_{i j}(t):=\left\langle\lambda(t),\left[f_{i}, f_{j}\right](x(t))\right\rangle .\right.
$$

Along an abnormal extremal, we have for every $t \in[0, T]$,

$$
h_{0}(t)=\text { constant }, \quad h_{i}(t)=0, \quad i=1, \ldots, m .
$$

Differentiating (2.4), one gets, almost everywhere on $[0, T]$,

$$
h_{i 0}(t)+\sum_{j=1}^{m} h_{i j}(t) u_{j}(t)=0, \quad i \in\{0, \ldots, m\} .
$$


Definition 2.4. Along an abnormal extremal $(x(\cdot), \lambda(\cdot), u(\cdot))$ of the system (2.1), the Goh matrix $G(t)$ at time $t \in[0, T]$ is the $m \times m$ skew-symmetric matrix given by

$$
G(t):=\left(h_{i j}(t)\right)_{1 \leqslant i, j \leqslant m} .
$$

Since $G(t)$ is skew-symmetric, rank $G(t)$ is even, and Equation (2.5) rewrites, almost everywhere on $[0, T]$,

$$
G(t) u(t)=b(t)
$$

with $b(t):=-\left(h_{i 0}(t)\right)_{1 \leqslant i \leqslant m}$.

Note that, if $G(t)$ is invertible, then $u(t)$ is uniquely determined by Equation (2.7). This only occurs for $m$ even.

If $m$ is odd, $G(t)$ is never invertible. However, a similar construction is derived as follows. Define

$$
\bar{G}(t):=\left(h_{i j}(t)\right)_{0 \leqslant i, j \leqslant m} .
$$

Since $\bar{G}(t)$ is skew-symmetric, the determinant of $\bar{G}(t)$ is the square of a polynomial $\bar{P}(t)$ in the $h_{i j}(t)$ with degree $(m+1) / 2$, called the Pfaffian (see of $\bar{G}(t)$ [6]). From Equation (2.5), $\bar{G}(t)$ is not invertible, and thus, along the extremal, $\bar{P}(t)=0$. After differentiation, one gets, almost everywhere on $[0, T]$,

$$
\left\{\bar{P}, h_{0}\right\}(t)+\sum_{i=1}^{m} u_{j}(t)\left\{\bar{P}, h_{j}\right\}(t)=0 .
$$

Define the $(m+1) \times m$ matrix $\widetilde{G}(t)$ as $G(t)$ augmented with the row $\left(\left\{\bar{P}, h_{j}\right\}(t)\right)_{1 \leqslant j \leqslant m}$, and the $(m+1)$-dimensional vector $\widetilde{b}(t)$ as $b(t)$ augmented with the coefficient $-\left\{\bar{P}, h_{0}\right\}(t)$. Then, from Equations (2.7) and (2.9), there holds, almost everywhere on $[0, T]$,

$$
\widetilde{G}(t) u(t)=\widetilde{b}(t) .
$$

If $\widetilde{G}(t)$ is of rank $m$, then $u(t)$ is uniquely determined by Equation (2.10).

These facts, combined with Remark 2.2, motivate the following definition.

Definition 2.5. If $m$ is even (resp. odd), a singular trajectory $x(\cdot)$ is said to be of minimal order if:

(i) $\dot{x}(t)=0$, for almost every $t \in I_{\text {dep }}(x(\cdot))$;

(ii) it admits an abnormal extremal lift such that, for almost every $t \in[0, T] \backslash I_{\text {dep }}$, rank $G(t)=m$ if $m$ is even, resp., rank $\widetilde{G}(t)=m$ if $m$ is odd.

On the opposite, for arbitrary $m$, a singular trajectory is said to be a Goh trajectory if it admits an abnormal extremal lift along which the Goh matrix is identically equal to 0.

TheOREm 2.6. Let $m<n$ be a positive integer. There exists an open and dense subset $O_{m+1}$ of $V F(M)^{m+1}$ so that, if the $(m+1)$-tuple $\left(f_{0}, \ldots, f_{m}\right)$ belongs to $O_{m+1}$, then every nontrivial singular trajectory of the associated control-affine system $\dot{x}(t)=f_{0}(x(t))+\sum_{i=1}^{m} u_{i}(t) f_{i}(x(t))$, is of minimal order and of corank one. In addition, for every integer $N$, the set $O_{m+1}$ can be chosen so that its complement has codimension greater than $N$.

COROLlaRY 2.7. With the notations of Theorem 2.0 and if $m \geqslant 2$, there exists an open and dense subset $O_{m+1}$ of $V F(M)^{m+1}$ so that every control-affine system defined with an $(m+1)$-tuple of $O_{m+1}$ does not admit nontrivial Goh singular trajectories. 
2.3. Minimizing singular trajectories. We keep here the notations of the previous sections. Consider the control-affine system

$$
\dot{x}(t)=f_{0}(x(t))+\sum_{i=1}^{m} u_{i}(t) f_{i}(x(t))
$$

and the quadratic cost given by

$$
C_{U, g}(T, u)=\frac{1}{2} \int_{0}^{T}\left(u(t)^{T} U(x(t)) u(t)+g(t, x(t))\right) d t,
$$

where $U \in \mathcal{S}_{m}^{+}(M)$ and $g \in C^{\infty}(\mathbb{R} \times M)$. Here, $\mathcal{S}_{m}^{+}(M)$ denotes the set of smooth mappings $x \mapsto U(x)$ on $M$, taking values in the set $\mathcal{S}_{m}^{+}$of $m \times m$ real positive definite matrices.

For $x_{0} \in M$ and $T>0$, define the optimal control problem

$$
\inf \left\{C_{U, g}(T, u) \mid E_{x_{0}, T}(u)=x\right\} .
$$

We next state two sets of genericity results, depending whether the cost or the control system is fixed.

\subsubsection{Genericity w.r.t. the control system, with a fixed cost.}

Proposition 2.8. Fix $U \in \mathcal{S}_{m}^{+}(M)$ and $g \in C^{\infty}(\mathbb{R} \times M)$. There exists an open and dense subset $O_{m+1}$ of $V F(M)^{m+1}$ such that every nontrivial singular trajectory of a control-affine system defined by an $(m+1)$-tuple of $O_{m+1}$ is strictly abnormal for the optimal control problem (2.13).

Corollary 2.7 together with Proposition 2.8 yields the next corollary.

Corollary 2.9. Fix $U \in \mathcal{S}_{m}^{+}(M)$ and $g \in C^{\infty}(\mathbb{R} \times M)$. Let $m \geqslant 2$ be an integer. There exists an open and dense subset $O_{m+1}$ of $V F(M)^{m+1}$ so that the optimal control problem (2.13) defined with an $(m+1)$-tuple of $O_{m+1}$ does not admit nontrivial minimizing singular trajectories.

REMARK 2.10. In both previous results, the set $O_{m+1}$ can be chosen so that its complement has arbitrary codimension.

2.3.2. Genericity w.r.t. the cost, with a fixed control system. We endow $\mathcal{S}_{m}^{+}(M)$ with the Whitney topology. An $(m+1)$ - tuple $\left(f_{0}, \ldots, f_{m}\right)$ of $V F(M)^{m+1}$ is said to verify the Lie Algebra Rank Condition if the Lie algebra generated by $f_{0}, \ldots, f_{m}$ is of dimension $n$ at every point of $M$.

Proposition 2.11. Fix $\left(f_{0}, \ldots, f_{m}\right) \in V F(M)^{m+1}$ so that the Lie Algebra Rank Condition is satisfied and the zero control $u \equiv 0$ is not singular. Let $g \in C^{\infty}(\mathbb{R} \times$ $M)$. Then, there exists an open and dense subset $\mathcal{A}_{m}$ of $\mathcal{S}_{m}^{+}(M)$ such that every nontrivial singular trajectory of the control-affine system associated to the $(m+1)$ tuple $\left(f_{0}, \ldots, f_{m}\right)$ is strictly abnormal for the optimal control problem (2.13) defined with $U \in \mathcal{A}_{m}$ and $g$.

Assuming that the zero control $u \equiv 0$ is not singular is a necessary hypothesis. Indeed, the fact that a control $u$ is singular is a property of the sole $(m+1)$-tuple $\left(f_{0}, \ldots, f_{m}\right)$ and is independent of the cost. On the other hand, every trajectory $x:=x\left(\cdot, x_{0}, 0\right)$ associated to the zero control is always the projection of the normal extremal $(x(\cdot), 0,-1,0)$ of any optimal control problem (2.13). As a consequence, if the zero control is singular, such a trajectory $x\left(\cdot, x_{0}, 0\right)$ cannot be strictly abnormal. 
In order to handle the case of a singular zero control, it is therefore necessary to consider more general quadratic costs such as

$$
C_{U, \alpha, g}(T, u)=\int_{0}^{T}\left(\frac{1}{2} u(t)^{T} U(x(t)) u(t)+\alpha(x(t))^{T} u(t)+g(t, x(t))\right) d t
$$

where $U \in \mathcal{S}_{m}^{+}(M), \alpha \in C^{\infty}\left(M, \mathbb{R}^{m}\right)$ and $g \in C^{\infty}(\mathbb{R} \times M)$.

Proposition 2.12. Fix $\left(f_{0}, \ldots, f_{m}\right) \in V F(M)^{m+1}$ satisfying the Lie Algebra Rank Condition and $g \in C^{\infty}(\mathbb{R} \times M)$. Then, there exists an open and dense subset $\mathcal{B}_{m}$ of $\mathcal{S}_{m}^{+}(M) \times C^{\infty}\left(M, \mathbb{R}^{m}\right)$ such that every nontrivial singular trajectory of the control-affine system associated to the $(m+1)$-tuple $\left(f_{0}, \ldots, f_{m}\right)$ is strictly abnormal for the optimal control problem (2.11)-(2.14) defined with $(U, \alpha) \in \mathcal{B}_{m}$ and $g$.

2.4. Driftless control-affine systems. Let $T$ be a positive real number. Consider the driftless control-affine system

$$
\dot{x}(t)=\sum_{i=1}^{m} u_{i}(t) f_{i}(x(t)),
$$

where $\left(f_{1}, \ldots, f_{m}\right)$ is an $m$-tuple of smooth vector fields on $M$, and the set of admissible controls $u=\left(u_{1}, \ldots, u_{m}\right)$ is an open subset of $L^{\infty}([0, T], \Omega)$.

For every trajectory $x(\cdot):=x\left(\cdot, x_{0}, u\right)$ of (2.1), define $I_{\text {dep }}(x(\cdot))$ as the closed subset of $[0, T]$

$$
I_{\mathrm{dep}}(x(\cdot)):=\left\{t \in[0, T] \mid \operatorname{rank}\left\{f_{1}(x(t)), \ldots, f_{m}(x(t))\right\}<m\right\} .
$$

TheOrem 2.13. Let $m \leqslant n$ be a positive integer. There exists an open and dense subset $O_{m}$ of $\operatorname{VF}(M)^{m}$ so that, if the $m$-tuple $\left(f_{1}, \ldots, f_{m}\right)$ belongs to $O_{m}$, then every trajectory $x(\cdot)$ of the associated driftless control-affine system $\dot{x}=\sum_{i=1}^{m} u_{i} f_{i}(x)$ verifies

$$
\dot{x}(t)=0, \text { for a.e. } t \in I_{\mathrm{dep}}(x(\cdot)) .
$$

In addition, for every integer $N$, the set $O_{m}$ can be chosen so that its complement has codimension greater than $N$.

2.4.1. Singular trajectories. Let $x(\cdot)$ be a singular trajectory, projection of an abnormal extremal $(x(\cdot), \lambda(\cdot))$. Similarly to the previous section, we define, for $t \in[0, T]$ and $i, j \in\{1, \ldots, m\}$,

$$
h_{i}(t):=\left\langle\lambda(t), f_{i}(x(t)\rangle, \quad h_{i j}(t):=\left\langle\lambda(t),\left[f_{i}, f_{j}\right](x(t))\right\rangle .\right.
$$

For every $t \in[0, T]$,

$$
h_{i}(t)=0, \quad i=1, \ldots, m
$$

Differentiating (2.16), one gets, almost everywhere on $[0, T]$,

$$
\sum_{j=1}^{m} h_{i j}(t) u_{j}(t)=0, \quad i \in\{1, \ldots, m\} .
$$

Definition 2.14. Along an abnormal extremal $(x(\cdot), \lambda(\cdot), u(\cdot))$ of the system 2.1), the Goh matrix $G(t)$ at time $t \in[0, T]$ is the $m \times m$ skew-symmetric matrix given by

$$
G(t):=\left(h_{i j}(t)\right)_{1 \leqslant i, j \leqslant m} .
$$


Since $G(t)$ is skew-symmetric, rank $G(t)$ is even, and Equation 2.17) rewrites, almost everywhere on $[0, T]$,

$$
G(t) u(t)=0
$$

Note that, if rank $G(t)=m-1$, one can deduce from (2.19) an expression for $u(t)$, up to time reparameterization. This only occurs for $m$ odd.

If $m$ is even, rank $G(t)$ is always smaller than $m-1$. However, a similar construction is derived as follows. The determinant of $G(t)$ is the square of the Pfaffian $P(t)$, and, along the extremal, $P(t) \equiv 0$. After differentiation, one gets, almost everywhere on $[0, T]$,

$$
\sum_{i=1}^{m} u_{j}(t)\left\{P, h_{j}\right\}(t)=0
$$

Define the $(m+1) \times m$ matrix $\widetilde{G}(t)$ as $G(t)$ augmented with the row $\left(\left\{P, h_{j}\right\}(t)\right)_{1 \leqslant j \leqslant m}$. Then, from Equations (2.19) and (2.20), there holds, almost everywhere on $[0, T]$,

$$
\widetilde{G}(t) u(t)=0 .
$$

If $\widetilde{G}(t)$ is of rank $m-1$, one can deduce from (2.21) an expression for $u(t)$, up to time reparameterization.

Definition 2.15. If $m$ is odd (resp. even), a singular trajectory $x(\cdot)$ is said to be of minimal order if:

(i) $\dot{x}(t)=0$, for almost every $t \in I_{\text {dep }}(x(\cdot))$;

(ii) it admits an abnormal extremal lift such that, for almost every $t \in[0, T] \backslash I_{\text {dep }}$, rank $G(t)=m-1$ if $m$ is odd, resp., rank $\widetilde{G}(t)=m-1$ if $m$ is even.

On the opposite, for arbitrary $m$, a singular trajectory is said to be a Goh trajectory if it admits an abnormal extremal lift along which the Goh matrix is identically equal to 0 .

THEOREM 2.16. Let $m$ be an integer such that $2 \leqslant m \leqslant n$. There exists an open and dense subset $O_{m}$ of $V F(M)^{m}$ so that, if the $m$-tuple $\left(f_{1}, \ldots, f_{m}\right)$ belongs to $O_{m}$, then every nontrivial singular trajectory of the associated driftless control-affine system $\dot{x}(t)=\sum_{i=1}^{m} u_{i}(t) f_{i}(x(t))$ is of minimal order and of corank one. In addition, for every integer $N$, the set $O_{m}$ can be chosen so that its complement has codimension greater than $N$.

Corollary 2.17. With the notations of Theorem 2.10 and if $m \geqslant 3$, there exists an open and dense subset $O_{m}$ of $V F(M)^{m}$ so that every driftless control-affine system defined with an $m$-tuple of $O_{m}$ does not admit nontrivial Goh singular trajectories.

2.4.2. Minimizing singular trajectories. Consider the optimal control problem associated to the driftless control-affine system

$$
\dot{x}(t)=\sum_{i=1}^{m} u_{i}(t) f_{i}(x(t)),
$$

with the quadratic cost given by

$$
C_{U, g}(T, u)=\frac{1}{2} \int_{0}^{T}\left(u(t)^{T} U(x(t)) u(t)+g(t, x(t))\right) d t
$$


where $U \in \mathcal{S}_{m}^{+}(M)$ and $g \in C^{\infty}(\mathbb{R} \times M)$.

For $x_{0} \in M$ and $T>0$, define the optimal control problem

$$
\inf \left\{C_{U, g}(T, u) \mid E_{x_{0}, T}(u)=x\right\} .
$$

We next state genericity results with respect to the control system, with a fixed cost.

Proposition 2.18. Fix $U \in \mathcal{S}_{m}^{+}(M)$ and $g \in C^{\infty}(\mathbb{R} \times M)$. There exists an open and dense subset $O_{m}$ of $V F(M)^{m}$ such that every nontrivial singular trajectory of a driftless control-affine system defined by an $m$-tuple of $O_{m}$ is strictly abnormal for the optimal control problem (2.24).

Corollary 2.17 together with Proposition 2.18 yields the next corollary.

Corollary 2.19. Fix $U \in \mathcal{S}_{m}^{+}(M)$ and $g \in C^{\infty}(\mathbb{R} \times M)$. Let $m \geqslant 3$ be an integer. There exists an open and densee subset $O_{m}$ of $V F(M)^{m}$ so that the optimal control problem (2.24) defined with an $m$-tuple of $O_{m}$ does not admit nontrivial minimizing singular trajectories.

REMARK 2.20. In both previous results, the set $O_{m}$ can be chosen so that its complement has arbitrary codimension.

We also have have a genericity result with respect to the cost, with a fixed control system.

Proposition 2.21. Fix $\left(f_{1}, \ldots, f_{m}\right) \in V F(M)^{m}$ so that the Lie Algebra Rank Condition is satisfied. Let $g \in C^{\infty}(\mathbb{R} \times M)$. Then, there exists an open and dense subset $\mathcal{A}_{m}$ of $\mathcal{S}_{m}^{+}(M)$ such that every nontrivial singular trajectory of the driftless control-affine system associated to the $m$-tuple $\left(f_{1}, \ldots, f_{m}\right)$ is strictly abnormal for the optimal control problem (2.24) defined with $U \in \mathcal{A}_{m}$ and $g$.

REMARK 2.22. In the driftless case, the control $u \equiv 0$ is always singular, but corresponds to a trivial trajectory. Therefore, in opposition to the control-affine case, it is not necessary to add the linear term $\alpha(x)^{T} u$ in the cost.

\section{Consequences.}

3.1. Regularity of the value function. Consider the optimal control problem (2.13), associated to the control-affine system (2.11) and the cost (2.12). The value function is defined by

$$
S_{x_{0}, T}(x):=\inf \left\{C_{U, g}(T, u) \mid E_{x_{0}, T}(u)=x\right\},
$$

for every $x \in \mathbb{R}^{n}$ (with, as usual, $\inf \emptyset:=-\infty$ ). We assume in the sequel that all data are analytic.

The regularity of $S_{x_{0}, T}$ is closely related to the existence of nontrivial minimizing singular trajectories starting from $x_{0}$. It is proved in [29] that, in the absence of minimizing singular trajectories, the value function is continuous and subanalytic (see e.g. [16] for a definition of a subanalytic function). For driftless control-affine systems and $g \equiv 0$, the value function coincides with the square of a sub-Riemannian distance (see [7] for an introduction to sub-Riemannian geometry). In particular, in this case, the value function is always continuous, but the trivial trajectory $x(\cdot) \equiv x_{0}$ is always minimizing and singular. Moreover, if there is no nontrivial minimizing singular trajectories, then the value function is subanalytic outside $x_{0}$ (see [3, (1). This situation holds for generic distributions of rank greater than or equal to three (see [5, 14]).

The results of Section 2.3 have the following consequence on the regularity of $S_{x_{0}, T}$.

Corollary 3.1. With the notations of Corollary 2.9, and if in addition the functions $g, U$, and the vector fields of the $(m+1)$-tuple in $O_{m+1}$ are analytic, then 
the associated value function $S_{x_{0}, T}$ is continuous and subanalytic on its domain of definition.

REMARK 3.2. If there exists a nontrivial minimizing trajectory, the value function may fail to be subanalytic or even continuous. For example, consider the control-affine system in $\mathbb{R}^{2}$ given by

$$
\dot{x}(t)=1+y(t)^{2}, \quad \dot{y}(t)=u(t),
$$

and the cost $C(T, u)=\int_{0}^{T} u(t)^{2} d t$. The trajectory $(x(t)=t, y(t)=0)$, associated to the control $u=0$, is a nontrivial minimizing singular trajectory, and the value function $S_{(0,0), T}$ has the asymptotic expansion, near the point $(T, 0)$,

$$
S_{(0,0), T}(x, y)=\frac{1}{4} \frac{y^{4}}{x-T}+\frac{y^{4}}{x-T} \exp \left(-\frac{y^{2}}{x-T}\right)+\text { o }\left(\frac{y^{4}}{x-T} \exp \left(-\frac{y^{2}}{x-T}\right)\right)
$$

(see [29] for details). Hence, it is not continuous, nor subanalytic, at the point $(T, 0)$.

In the driftless control-affine case, by using results of Section 2.4.2, we derive the following similar consequence.

COROLlary 3.3. With the notations of Corollary 2.19, and if in addition the functions $g, U$, and the vector fields of the $m$-tuple in $O_{m}$ are analytic, then the associated value function $S_{x_{0}, T}$ is subanalytic outside $x_{0}$.

3.2. Regularity of viscosity solutions of Hamilton-Jacobi equations. Assume that the assumptions of the previous subsection hold. It is standard (see [15, 17) that the value function $v(t, x)=S_{x_{0}, t}(x)$ is a viscosity solution of the Hamilton-Jacobi equation

$$
\frac{\partial v}{\partial t}+\mathcal{H}\left(x, \frac{\partial v}{\partial x}\right)=g(t, x)
$$

where $\mathcal{H}(x, p)=\left\langle p, f_{0}(x)\right\rangle+\frac{1}{2} \sum_{i, j=1}^{m}\left(U^{-1}(x)\right)_{i j}\left\langle p, f_{i}(x)\right\rangle\left\langle p, f_{j}(x)\right\rangle$.

Conversely, the viscosity solution of (3.2) with analytic Dirichlet-type conditions is subanalytic, as soon as the corresponding optimal control does not admit minimizing singular trajectories (see [31]). Using the results of the previous sections, this situation holds generically if $m \geqslant 2$ (and, similarly for driftless control-affine systems, if $m \geqslant 3$ ).

As a consequence, the analytic singular set $\operatorname{Sing}(v)$ of the viscosity solution $v$, i.e., the subset of $\mathbb{R}^{n}$ where $v$ is not analytic, is a (subanalytic) stratified manifold of codimension greater than or equal to one (see [28] for more details on the subject). Since $\operatorname{Sing}(v)$ is also the locus where characteristic curves intersect, the abovementioned property turns out to be instrumental for the global convergence of numerical schemes for Equation (3.2) (see [15]). Indeed, the analytic singular set must be as "nice" as possible in order to integrate energy functions on the set of characteristic curves.

3.3. Applications to stabilization and motion planning. For a driftless control-affine system verifying the Lie Algebra Rank Condition, there exist general stabilizing strategies stemming from dynamic programming. As usual, the stabilizing feedback is computed using the gradient of the value function $S$ for a suitable optimal control problem. Of course this is only possible outside the singular set $\operatorname{Sing}(S)$, and one must device another construction for the feedback on $\operatorname{Sing}(S)$. Let us mention two such strategies, the first one providing an hybrid feedback (see 22 ), and the second one a smooth repulsive stabilizing (SRS) feedback (see [23, 24]). Both strategies 
crucially rely on the fact that $\operatorname{Sing}(S)$ is a stratified manifold of codimension greater than or equal to one.

As seen before, the latter fact holds generically in the analytic category for $m \geqslant 3$.

On the other hand, the absence of singular minimizing trajectories is the basic requirement for the convergence of usual algorithms in optimal control (such as direct or indirect methods, see e.g. [8, 20]). We have proved that this situation holds generically for control-affine systems if $m \geqslant 2$, and for driftless control-affine systems if $m \geqslant 3$.

As a final application, consider a driftless control-affine system verifying the Lie Algebra Rank Condition. According to Proposition 2.21, it is possible to choose a (generic) cost function $C_{U, g}$ such that all singular trajectories are strictly abnormal. Combining that fact with 225, Theorem 1.1], we deduce that there exists a dense subset $N$ of $\mathbb{R}^{n}$ such that every point of $N$ is reached by a unique minimizing trajectory, which is moreover nonsingular. As a consequence, a shooting method with target in $N$ will converge. That fact may be used for solving (at least approximately) motion planning problems.

\section{Proofs of the results.}

4.1. Proof of Theorem 2.1 and of Theorem 2.13. Every trajectory of the control-affine system $\dot{x}=f_{0}(x)+\sum_{i=1}^{m} u_{i} f_{i}(x)$ is also a trajectory of the driftless control system $\dot{x}=\sum_{i=0}^{m} u_{i} f_{i}(x)$, with $u_{0} \equiv 1$. Therefore, Theorem 2.1 follows from Theorem 2.13, whose proof is provided next.

Let $x(\cdot)=x\left(\cdot, x_{0}, u\right)$ be a trajectory of the driftless control system $\dot{x}=\sum_{i=1}^{m} u_{i} f_{i}(x)$, with $2 \leqslant m \leqslant n$. Consider the set $I_{\text {dep }}(x(\cdot))$ defined by (2.2). We argue by contraposition, and assume that $I_{\mathrm{dep}}(x(\cdot))$ contains a subset $I$ of positive measure such that $\dot{x}(t) \neq 0$ for $t \in I$. Since Lebesgue points of $u$ are of full Lebesgue measure, we assume that $u$ is continuous on $I$.

Up to considering a subset of $I$, and relabeling the $f_{i}$ 's, we assume that, for every $t \in I$ :

(i) there exists $1 \leqslant k<m$ such that

$$
\operatorname{rank}\left\{f_{1}(x(t)), \ldots, f_{m}(x(t))\right\}=k ;
$$

(ii) $f_{1}(x(t)), \ldots, f_{k}(x(t))$ are linearly independent, and thus, there exist real numbers $\alpha_{i}^{j}(t), i=1, \ldots, k, j=k+1, \ldots, m$, such that

$$
f_{j}(x(t))=\sum_{i=1}^{k} \alpha_{i}^{j}(t) f_{i}(x(t)), \quad j=k+1, \ldots, m .
$$

Therefore, $\dot{x}(t)=\sum_{i=1}^{k} \delta_{i}(t) f_{i}(x(t))$, where $\delta_{i}(t):=u_{i}(t)+\sum_{j=k+1}^{m} \alpha_{i}^{j}(t) u_{j}(t)$;

(iii) $\delta_{1}(t) \neq 0$.

REMARK 4.1. Up to reducing $I$, we furthermore assume that $I$ is contained in an open interval $\mathcal{I}$ on which $\operatorname{rank}\left\{f_{1}(x(t)), \ldots, f_{k}(x(t))\right\}=k$.

Set $\operatorname{ad}^{0} g(h)=h$, where $g, h \in \operatorname{VF}(M)$, and $\operatorname{ad}^{k} g(h)=\left[g, \operatorname{ad}^{k-1} g(h)\right]$ for $k \geqslant 1$. The length of the iterated Lie bracket $\left[f_{i_{1}},\left[f_{i_{2}},\left[\cdots, f_{i_{k}}\right] \cdots\right]\right.$ of $f_{1}, \ldots, f_{m}$ is the integer $k$.

Proposition 4.2. Let $N$ be a positive integer. There exists a subset $J_{N} \subset I$ of positive measure such that, for every $t \in J_{N}$, and every $\ell \in\{1, \ldots, N\}$,

$$
\delta_{1}(t)^{\ell-1} \operatorname{ad}^{\ell-1} f_{1}\left(f_{m}\right)(x(t))=h_{t}^{\ell}(x(t))+R_{t}^{\ell}(x(t)),
$$


where

- $h_{t}^{\ell}(x(t)) \in \operatorname{Span}\left\{f_{1}(x(t)), \ldots, f_{k}(x(t))\right\}$,

- $R_{t}^{\ell}$ is a linear combination of iterated Lie brackets of $f_{1}, \ldots, f_{m}$, of length smaller than $\ell-1$, and of iterated Lie brackets of $f_{1}, \ldots, f_{k}$, of length smaller than or equal to $\ell$.

Proof. For $t \in I$, let $F_{t} \in V F(M)$ be the vector field defined by

$$
F_{t}(x):=\sum_{i=1}^{k} \delta_{i}(t) f_{i}(x) .
$$

Notice that $\dot{x}(t)=F_{t}(x(t))$, for $t \in I$. For the argument of Proposition 4.2, we need the following lemma.

Lemma 4.3. Consider a set $J \subset I$ of positive measure and $h \in V F(M)$ so that $h(x(t)) \in \operatorname{Span}\left\{f_{1}(x(t)), \ldots, f_{k}(x(t))\right\}$ on $J$, i.e., for every $t \in J$, there exist real numbers $\beta_{i}(t), i=1, \ldots, k$, such that

$$
h(x(t))=\sum_{i=1}^{k} \beta_{i}(t) f_{i}(x(t)) .
$$

For $t \in J$, define $g_{t} \in V F(M)$ by

$$
g_{t}(x):=h(x)-\sum_{i=1}^{k} \beta_{i}(t) f_{i}(x) .
$$

Then, there exists a set $J^{\prime} \subset J$ of positive measure such that

$$
\left[F_{t}, g_{t}\right](x(t)) \in \operatorname{Span}\left\{f_{1}(x(t)), \ldots, f_{k}(x(t))\right\} \quad \text { on } J^{\prime} \text {. }
$$

Proof. [Proof of Lemma 4.3] Using Remark 4.1, there exist $e_{j} \in V F(M), k+1 \leqslant$ $j \leqslant n$, so that, for every $t \in \mathcal{I}$, the vectors $f_{1}(x(t)), \ldots, f_{k}(x(t)), e_{k+1}(x(t)), \ldots$, $e_{n}(x(t))$ span $T_{x(t)} M$. Thus, there exist $n$ smooth functions $b_{i}, 1 \leqslant i \leqslant n$, defined on $M$, such that

$$
h(x)=\sum_{i=1}^{k} b_{i}(x) f_{i}(x)+\sum_{i=k+1}^{n} b_{i}(x) e_{i}(x),
$$

for $x$ in an open neighborhood of $x(\mathcal{I})$. For $i=1, \ldots, n$, define $\beta_{i}(t):=b_{i}(x(t))$, for $t \in \mathcal{I}$ (this notation is consistent with (4.2)). The $\beta_{i}^{\prime} s$ are absolutely continuous on $\mathcal{I}$ and differentiable everywhere on $J$. For $i=k+1, \ldots, n$, there holds $\beta_{i} \equiv 0$ on $J$ and therefore, it follows that $\dot{\beta}_{i} \equiv 0$ on a subset $J^{\prime} \subset J$ of full measure (cf. 26, Lemma p. 177]).

For $t \in J$, using that $g_{t}(x(t))=0$, and $F_{t}(x(t))=\dot{x}(t)$, it holds

$$
\begin{aligned}
{\left[F_{t}, g_{t}\right](x(t)) } & =d g_{t} \circ F_{t}(x(t)) \\
& =\sum_{i=1}^{k}\left(d b_{i}(x(t)) \cdot \dot{x}(t)\right) f_{i}(x(t))+\sum_{i=k+1}^{n}\left(d b_{i}(x(t)) \cdot \dot{x}(t)\right) e_{i}(x(t)) \\
& =\sum_{i=1}^{k} \dot{\beta}_{i}(t) f_{i}(x(t))+\sum_{i=k+1}^{n} \dot{\beta}_{i}(t) e_{i}(x(t)) .
\end{aligned}
$$


On $J^{\prime}$, the second sum of the right-hand side of the last equation vanishes, and the lemma follows.

Applying Lemma 4.3 to $h=f_{m}$ and $J=I$, we get

$$
\left[F_{t}, g_{t}^{1}\right](x(t)) \in \operatorname{Span}\left\{f_{1}(x(t)), \ldots, f_{k}(x(t))\right\} \quad \text { on } J_{1},
$$

where $J_{1} \subset I$ and $g_{t}^{1}:=f_{m}-\sum_{i=1}^{k} \alpha_{i}^{m}(t) f_{i}$.

Set $h_{t}^{1}=\left[F_{t}, g_{t}^{1}\right]$. We next iterate the above procedure, for $1 \leqslant \ell \leqslant N$. Assume that the vector fields $h_{t}^{\ell}, g_{t}^{\ell}$, and the set $J_{\ell}$ of positive measure are defined, such that $h_{t}^{\ell}(x(t)) \in \operatorname{Span}\left\{f_{1}(x(t)), \ldots, f_{k}(x(t))\right\}$ on $J_{\ell}$. For every $t \in J_{\ell}$, let $\beta_{i}^{\ell}(t), i=1, \ldots, k$, be the real numbers such that

$$
h_{t}^{\ell}(x(t))=\sum_{i=1}^{k} \beta_{i}^{\ell}(t) f_{i}(x(t)),
$$

and define $g_{t}^{\ell+1} \in V F(M)$ by $g_{t}^{\ell+1}:=h_{t}^{\ell}-\sum_{i=1}^{k} \beta_{i}^{\ell}(t) f_{i}$. Set $h_{t}^{\ell+1}:=\left[F_{t}, g_{t}^{\ell+1}\right]$. Applying Lemma 1.3, there exists a subset $J_{\ell+1} \subset J_{\ell}$ of positive measure such that $h_{t}^{\ell+1}(x(t)) \in \operatorname{Span}\left\{f_{1}(x(t)), \ldots, f_{k}(x(t))\right\}$ on $J_{\ell+1}$.

For $t \in J_{N}$, and for $\ell=1, \ldots, N$, we express $h_{t}^{\ell}(x(t))$ using iterated Lie brackets of $f_{1}, \ldots, f_{m}$, and an easy induction yields (4.1).

Combining Proposition 4.2 with routine transversality arguments (see for instance [12] and [14]), it follows that the $(m+1)$-tuple $\left(f_{0}, \ldots, f_{m}\right)$ belongs to a closed subset of $V F(M)^{m+1}$ of codimension greater than or equal to $N$. Theorem 2.13 follows.

REMARK 4.4. The fact that $f_{1}(x(t)) \neq 0$ is essential in order to derive, from (4.1), an infinite number of independent relations, and then to apply the abovementioned transversality arguments.

4.2. Proof of Theorem 2.6. The minimal order and corank one properties are proved separately in the following lemmas.

LEMMA 4.5. There exists an open and dense subset $O_{m+1}^{1}$ of $V F(M)^{m+1}$ so that, if the $(m+1)$-tuple $\left(f_{0}, \ldots, f_{m}\right)$ belongs to $O_{m+1}^{1}$, then every singular trajectory of the associated control-affine system $\dot{x}(t)=f_{0}(x(t))+\sum_{i=1}^{m} u_{i}(t) f_{i}(x(t))$ is of minimal order. In addition, for every integer $N$, the set $O_{m+1}^{1}$ can be chosen so that its complement has codimension greater than $N$.

LEMma 4.6. There exists an open and dense subset $O_{m+1}$ of $O_{m+1}^{1}$ so that, if the $(m+1)$-tuple $\left(f_{0}, \ldots, f_{m}\right)$ belongs to $O_{m+1}$, then every nontrivial singular trajectory of the associated control-affine system $\dot{x}(t)=f_{0}(x(t))+\sum_{i=1}^{m} u_{i}(t) f_{i}(x(t))$ is of corank one. In addition, for every integer $N$, the set $O_{m+1}^{2}$ can be chosen so that its complement has codimension greater than $N$.

The conclusion of Theorem 2.6 with the set $O_{m+1}$ whose existence is stated above.

4.2.1. Proof of Lemma 4.5. From Theorem 2.1, there exists an open and dense subset $O_{m+1}^{11}$ of $V F(M)^{m+1}$ such that, if $\left(f_{0}, \ldots, f_{m}\right) \in O_{m+1}^{11}$, then every trajectory $x(\cdot)$ of $\dot{x}=f_{0}(x)+\sum_{i=1}^{m} u_{i} f_{i}(x)$ verifies Item (i) of Definition 2.5 .

It is therefore enough to show the existence of an open and dense subset $O_{m+1}^{12}$ of $V F(M)^{m+1}$ such that, if $\left(f_{0}, \ldots, f_{m}\right) \in O_{m+1}^{12}$, then every singular trajectory $x(\cdot)$ of $\dot{x}=f_{0}(x)+\sum_{i=1}^{m} u_{i} f_{i}(x)$ verifies Item (ii) of Definition 2.5. Then, by choosing $O_{m+1}^{1}:=O_{m+1}^{11} \cap O_{m+1}^{12}$, the conclusion of Lemma 4.5 follows.

Consider a singular trajectory $x(\cdot):=x\left(\cdot, x_{0}, u\right)$ of $\dot{x}=f_{0}(x)+\sum_{i=1}^{m} u_{i} f_{i}(x)$, admitting an abnormal extremal $(x(\cdot), \lambda(\cdot))$. Assume that there exists $J \subset[0, T] \backslash$ $I_{\text {dep }}(x(\cdot))$ of positive measure such that $G(t)$ is not of rank $m$ if $m$ is even, respectively, 
$\widetilde{G}(t)$ is not of rank $m$ if $m$ is odd. We will show that the $(m+1)$-tuple $\left(f_{0}, \ldots, f_{m}\right)$ belongs to a subset of arbitrary codimension in $V F(M)^{m+1}$ whose complement contains an open and dense subset.

Note that, on $[0, T] \backslash I_{d e p}(x(\cdot))$, the vector fields $f_{0}(x(t)), \ldots, f_{m}(x(t))$ are linearly independent. The remaining part of the argument consists of reformulating the problem in order to follow the chain of arguments in the proof of 114. Theorem $2.4]$ concerning the case of everywhere linearly independent vector fields. For that purpose, we distinguish the cases $m$ even and $m$ odd.

Assume first that $m$ is even. As in (2.8), define, for $t \in J, \bar{G}(t):=\left(h_{i j}(t)\right)_{0 \leqslant i, j \leqslant m}$. From (2.7), we have, for $t \in J$,

$$
\bar{G}(t)=\left(\begin{array}{cc}
0 & (G(t) u(t))^{T} \\
-G(t) u(t) & G(t)
\end{array}\right) .
$$

Since the ranks of both $\bar{G}(t)$ and $G(t)$ are even, they must be equal, for $t \in J$, and hence, the rank of $\bar{G}(t)$ is smaller than $m$ on $J$. This is exactly the starting point of the proof of [14, Lemma 3.8]. The machinery of [14 then applies and we deduce that the $(m+1)$-tuple $\left(f_{0}, \ldots, f_{m}\right)$ belongs to a subset of arbitrary codimension in $V F(M)^{m+1}$ whose complement contains an open and dense subset $O_{m+1}^{2}$ of $V F(M)^{m+1}$.

Assume next that $m$ is odd. Define the $(m+2) \times(m+1)$ matrix $\widehat{G}(t)$ as $\bar{G}(t)$ augmented in the last row with $\left(\left\{\bar{P}, h_{j}\right\}(t)\right)_{0 \leqslant j \leqslant m}$.

LEMma 4.7. With the notations above, $\operatorname{rank} \widehat{G}(t) \leqslant \operatorname{rank} \widetilde{G}(t)+1$.

Proof. It amounts to show that, $\xi \in \operatorname{ker} \widetilde{G}(t)$ implies $(0, \xi) \in \operatorname{ker} \widehat{G}(t)$. This follows from the fact that if $\widetilde{G}(t) \xi=0$, then $G(t) \xi=0$, and thus $\xi$ is orthogonal to the range of $G(t)$ since $G(t)$ is skew-symmetric. $\square$

Using Lemma 4.7, the rank of $\widehat{G}(t)$ is less than $m+1$ on $J$. This is exactly the starting point of the proof of [14, Lemma 3.9]. The machinery of [14] then applies and we deduce that the $(m+1)$-tuple $\left(f_{0}, \ldots, f_{m}\right)$ belongs to a subset of arbitrary codimension in $V F(M)^{m+1}$ whose complement contains an open and dense subset $O_{m+1}^{12}$ of $V F(M)^{m+1}$.

4.2.2. Proof of Lemma 4.6. We argue by contraposition. Consider a nontrivial singular trajectory $x(\cdot):=x\left(\cdot, x_{0}, u\right)$ of $\dot{x}=f_{0}(x)+\sum_{i=1}^{m} u_{i} f_{i}(x)$, with $\left(f_{0}, \ldots, f_{m}\right) \in$ $O_{m+1}^{1}$. Assume that $x(\cdot)$ admits two abnormal extremal lifts $\left(x(\cdot), \lambda^{[1]}(\cdot)\right)$ and $\left(x(\cdot), \lambda^{[2]}(\cdot)\right)$ such that, for some $t_{0} \in[0, T], \lambda^{[1]}\left(t_{0}\right)$ and $\lambda^{[2]}\left(t_{0}\right)$ are linearly independent. By linearity, $\lambda^{[1]}(\cdot)$ and $\lambda^{[2]}(\cdot)$ are linearly independent everywhere on $[0, T]$. Since $x(\cdot)$ is nontrivial, it follows from Remark 2.3 that there exists a nonempty subinterval $J$ of $[0, T] \backslash I_{d e p}(x(\cdot))$. We are now in a position to exactly follow the arguments of [14] corresponding to the corank one property, i.e., [14, Lemma 4.4].

4.3. Proof of Theorem 2.16. We start with the proof of the statement dealing with the minimal order property.

From Theorem 2.13, there exists an open and dense subset $O_{m}^{1}$ of $V F(M)^{m}$ such that, if $\left(f_{1}, \ldots, f_{m}\right) \in O_{m}^{1}$, then every trajectory $x(\cdot)$ of $\dot{x}=\sum_{i=1}^{m} u_{i} f_{i}(x)$ verifies Item (i) of Definition 2.15.

It is therefore enough to show the existence of an open and dense subset $O_{m}^{2}$ of $V F(M)^{m}$ such that, if $\left(f_{1}, \ldots, f_{m}\right) \in O_{m}^{2}$, then every singular trajectory $x(\cdot)$ of $\dot{x}=$ $\sum_{i=1}^{m} u_{i} f_{i}(x)$ verifies Item (ii) of Definition 2.15. Then, by choosing $O_{m}:=O_{m}^{1} \cap O_{m}^{2}$, the statement dealing with the minimal order property in Theorem 2.16 follows. 
Consider a singular trajectory $x(\cdot):=x\left(\cdot, x_{0}, u\right)$ of $\dot{x}=\sum_{i=1}^{m} u_{i} f_{i}(x)$ admitting an abnormal extremal $(x(\cdot), \lambda(\cdot))$. Assume that there exists $J \subset[0, T] \backslash I_{\text {dep }}(x(\cdot))$ of positive measure such that $G(t)$ is not of rank $m-1$ if $m$ is odd, respectively, $\widetilde{G}(t)$ is not of rank $m-1$ if $m$ is even. Following exactly the proofs of Lemmas 3.8 and 3.9 in [14], the $m$-tuple $\left(f_{1}, \ldots, f_{m}\right)$ belongs to a subset of arbitrary codimension in $V F(M)^{m}$ whose complement contains an open and dense subset.

We proceed similarly for an argument of the statement dealing with the corank one property.

4.4. Proofs of Propositions 2.8 and 2.18. We only treat the control-affine case, the argument for the driftless control-affine case being identical. We argue by contraposition. Consider a nontrivial singular trajectory $x(\cdot):=x\left(\cdot, x_{0}, u\right)$ of $\dot{x}=f_{0}(x)+\sum_{i=1}^{m} u_{i} f_{i}(x)$, with $\left(f_{0}, \ldots, f_{m}\right) \in V F(M)^{m+1}$. Assume that $x(\cdot)$ admits on the one part a normal extremal lift $\left(x(\cdot), \lambda^{[n]}(\cdot)\right)$ and on the other part an abnormal extremal lift $\left(x(\cdot), \lambda^{[a]}(\cdot)\right)$.

Let us introduce some notations. For $k \in \mathbb{N}$, let $L=l_{1} \cdots l_{k}$ be a multi-index of $\{0, \ldots, m\}$. The length of $L$ is $|L|=k$ and $f_{L}$ is the vector field defined by

$$
f_{L}:=\left[\left[\ldots\left[f_{l_{1}}, f_{l_{2}}\right], \ldots\right], f_{l_{k}}\right] .
$$

A multi-index $L=j l \cdots l$ with $k$ consecutive occurrences of the index $l$ is denoted as $L=j l^{k}$.

For every multi-index $L$ of $\{0, \ldots, m\}$ and $t \in[0, T]$, set

$$
h_{L}^{[n]}(t)=\left\langle\lambda^{[n]}(t), f_{L}(x(t))\right\rangle \text {, and } h_{L}^{[a]}(t)=\left\langle\lambda^{[a]}(t), f_{L}(x(t))\right\rangle .
$$

After time differentiation, we have on $[0, T]$,

$$
\begin{aligned}
\frac{d}{d t} h_{L}^{[n]}(t) & =\sum_{l=1}^{m} u_{l}(t) h_{L l}^{[n]}(t), \\
\frac{d}{d t} h_{L}^{[a]}(t) & =\sum_{l=1}^{m} u_{l}(t) h_{L l}^{[a]}(t) .
\end{aligned}
$$

Recall that, according to the Pontryagin Maximum Principle, there holds

$$
u(t)=\left(\begin{array}{c}
u_{1}(t) \\
\vdots \\
u_{m}(t)
\end{array}\right)=U(x(t))^{-1}\left(\begin{array}{c}
h_{1}^{[n]}(t) \\
\vdots \\
h_{m}^{[n]}(t)
\end{array}\right),
$$

and, for every $t \in[0, T]$,

$$
h_{0}^{[a]}(t)=\text { constant, } h_{l}^{[a]}(t)=0,
$$

for every $l \in\{1, \ldots, m\}$, and $t \in[0, T]$. Since the trajectory $x(\cdot)$ is nontrivial, there exists an open interval $J \subset[0, T]$ and $i \in\{0, \ldots, m\}$ such that $u_{i}(\cdot) f_{i}(x(\cdot))$ is never vanishing (with the convention $u_{0} \equiv 1$ ). Fix $j \in\{0, \ldots, m\} \backslash\{i\}$. Differentiating $s$ times (with $s \geqslant 1$ ) the relation $h_{j}^{[a]}(t)=$ constant with respect to $t \in J$, one gets, by using (4.4), (4.5), and (4.6), that

$$
0=\frac{d^{s}}{d t^{s}} h_{j}^{[a]}(t)=\left(u_{i}(t)\right)^{s} h_{j i^{s}}^{[a]}(t)+R_{s}(t),
$$


where $R_{s}(t)$ is polynomial in $h_{L}^{[n]}(t)$ and $h_{K}^{[a]}(t),|L| \leqslant s,|K| \leqslant s+1$, with $K$ different from $j i^{s}$ and $i j i^{s-1}$. Fix $t \in J$. Since $u_{i}(t) \neq 0$ and $f_{i}(x(t)) \neq 0$, we are in a position to apply routine transversality arguments. It follows that the $(m+1)$-tuple $\left(f_{0}, \ldots, f_{m}\right)$ belongs to a closed subset of $V F(M)^{m+1}$ of arbitrary codimension. Proposition 2.8 follows.

4.5. Proofs of Propositions 2.11, 2.12, and 2.21. We first prove Proposition 2.12 and argue by contraposition. Consider a nontrivial singular trajectory $x(\cdot):=$ $x\left(\cdot, x_{0}, u\right)$ of $\dot{x}=f_{0}(x)+\sum_{i=1}^{m} u_{i} f_{i}(x)$. Assume that $x(\cdot)$ admits on the one part a normal extremal lift $\left(x(\cdot), \lambda^{n}(\cdot)\right)$ and on the other part an abnormal extremal lift $\left(x(\cdot), \lambda^{[a]}(\cdot)\right)$.

From the Pontryagin Maximum Principle, there holds, for $l=1, \ldots, m$,

$$
u_{l}(t)=\sum_{p=1}^{m} Q^{l p}(x(t)) \beta_{p}(x(t)), \quad \beta_{p}(x(t)):=h_{p}^{[n]}(t)-\alpha_{p}(x(t)),
$$

where the $Q^{l p}(x)$ and the $\alpha_{p}(x)$ are respectively the coefficients of $U^{-1}(x)$ and of $\alpha(x)$. Note that the $u_{l}$ 's are smooth functions of the time.

Since the trajectory $x(\cdot)$ is nontrivial, there exists an open interval $J \subset[0, T]$ such that $\dot{x}$ is never vanishing on $J$ and one of the two following cases holds.

Case 1: $u \equiv 0$ on $J$.

In that case, $\dot{x}(t)=f_{0}(x(t))$ for $t \in J$, and $f_{0}(x(\cdot))$ is never vanishing on $J$. Moreover, for $p=1, \ldots, m, \beta_{p} \equiv 0$ on $J$, i.e. $\alpha_{p}(x(t))=h_{p}^{[n]}(t)$ for $t \in J$. By differentiating the latter relation with respect to the time, we deduce that, for all $N \geqslant 0, t \in J$ and $p=1, \ldots, m$,

$$
L_{f_{0}}^{N} \alpha_{p}(x(t))=L_{f_{0}}^{N} h_{p}^{[n]}(x(t)),
$$

where $L_{f_{0}}$ denotes the Lie derivative with respect to the vector field $f_{0}$. Applying routine transversality arguments, it follows that $\alpha$ belongs to a closed subset of $C^{\infty}\left(M, \mathbb{R}^{m}\right)$ of arbitrary codimension.

Case 2: $u$ is never vanishing on $J$.

Using (2.4) and the Lie Algebra Rank Condition, there exist a multi-index $L$, an index $j_{0} \in\{0, \ldots, m\}$, and a subinterval of $J$ (still denoted $J$ ), such that

$$
h_{L}^{[a]}(t)=\text { constant, and } h_{L j_{0}}^{[a]}(t) \neq 0,
$$

for every $t \in J$. Differentiating $h_{L}^{[a]}$ on $J$, one gets

$$
\begin{aligned}
0=\frac{d}{d t} h_{L}^{[a]}(t) & =h_{L 0}^{[a]}(t)+\sum_{l=1}^{m} u_{l}(t) h_{L l}^{[a]}(t) \\
& =h_{L 0}^{[a]}(t)+\sum_{1 \leqslant l \leqslant p \leqslant m} c_{l p}(t) Q^{l p}(x(t)),
\end{aligned}
$$

where $c_{l l}(t):=\beta_{l}(t) h_{L l}^{[a]}(t)$, and $c_{l p}(t):=\beta_{p}(t) h_{L l}^{[a]}(t)+\beta_{l}(t) h_{L p}^{[a]}(t)$ if $l<p$.

Lemma 4.8. Up to reducing the interval $J$, there exist indices $j$ and $l$ in $\{1, \ldots, m\}$ such that $c_{l j}(t)$ or $c_{j l}(t)$ is never vanishing on $J$.

Proof. If $j_{0}=0$, then $h_{L 0}^{[a]}(t) \neq 0$, and it follows from (4.9) that there exist $l, j \in\{1, \ldots, m\}$ such that $c_{l j}(t) \neq 0$. Otherwise, take $j:=j_{0}$. In that case, one 
of the $\beta_{p}$ 's does not vanish on $J$ since $u$ is not zero. First, assume that $\beta_{j}(t)$ is not identically equal to zero on $J$; then, up to reducing $J, c_{j j}(t)$ is never vanishing on $J$. Otherwise, there exists $l \neq j$ such that, up to reducing $J, \beta_{l}$ is never vanishing on $J$ and thus similarly for $c_{l j}$ (or $c_{j l}$ ).

For $t \in J$, let $F_{t} \in V F(M)$ be the vector field defined by

$$
F_{t}(x):=f_{0}(x)+\sum_{i=1}^{m} u_{i}(t) f_{i}(x) .
$$

Notice that $F_{t}(x(t))=\dot{x}(t) \neq 0$. For all $N \geqslant 0$ and $t \in J$, we get, by taking the $(N+1)$-th time derivative of $h_{L}^{[a]}$ on $J$,

$$
0=\frac{d^{N+1}}{d t^{N+1}} h_{L}^{[a]}(t)=c_{l j}(t) L_{F_{t}}^{N} Q^{j l}(x(t))+R_{N}(t)
$$

where $R_{N}(t)$ is a linear combination of $L_{F_{t}}^{s} Q^{p i}(x(t))$, with $s \leqslant N, p \leqslant i$ in $\{1, \ldots, m\}$ and $s<N$ if $(p, i)=(j, l)$, and of $L_{f_{r}}^{s} Q^{p i}(x(t))$, with $s<N, p \leqslant i$ in $\{1, \ldots, m\}$, and $r \in\{0, \ldots, m\}$. Applying routine transversality arguments, it follows that $(U, \alpha)$ belongs to a closed subset of $\mathcal{S}_{m}^{+}(M) \times C^{\infty}\left(M, \mathbb{R}^{m}\right)$ of arbitrary codimension. Proposition 2.12 is proved.

To show Propositions 2.11 and 2.21, simply notice that the argument of Case 2 with $\alpha=0$ applies with suitable modifications.

\section{REFERENCES}

[1] A. Agrachev, A. Sarychev, Strong minimality of abnormal geodesics for 2-distributions, J. Dyn. Cont. Syst. 1, 2, 1995.

[2] A. Agrachev, A. Sarychev, On abnormal extremals for Lagrange variational problems, J. Math. Syst. Estim. Cont. 8, 1, 1998, 87-118.

[3] A. Agrachev, Compactness for sub-Riemannian length minimizers and subanalyticity, Rend. Semin. Mat. Torino 56, 4, 1998, 1-12.

[4] A. Agrachev, A. Sarychev, Sub-Riemannian metrics: minimality of abnormal geodesics versus subanalyticity, ESAIM Control Optim. Calc. Var. 4, 1999, 377-403.

[5] A. Agrachev, J.-P. Gauthier, On subanalyticity of Carnot-Carathéodory distances, Ann. Inst. H. Poincaré Anal. Non Linéaire 18, 3, 2001.

[6] E. Artin, Geometric Algebra, John Wiley and Sons Inc, 1988.

[7] A. Bellaïche, Tangent space in sub-Riemannian geometry, in Sub-Riemannian geometry, Birkhäuser, 1996.

[8] J.T. Betts, Practical methods for optimal control using nonlinear programming, Advances in Design and Control, SIAM, Philadelphia, PA, 2001.

[9] G. A. Bliss, Lectures on the calculus of variations, U. of Chicago Press, 1946.

[10] B. Bonnard, M. Chyba, The role of singular trajectories in control theory, Math. \& Appl. 40, Springer-Verlag, 2003.

[11] B. Bonnard, I. Kupka, Théorie des singularités de l'application entrée/sortie et optimalité des trajectoires singulières dans le problème du temps minimal, Forum Math. 5, 1993.

[12] B. Bonnard, I. Kupka, Generic properties of singular trajectories, Ann. Inst. H. Poincaré Anal. Non Linéaire 14, 2, 1997.

[13] Y. Chitour, F. Jean, E. Trélat, Propriétés génériques des trajectoires singulières, Comptes Rendus Math. 337, 1, 2003, pp. 49-52.

[14] Y. Chitour, F. Jean, E. Trélat, Genericity results for singular curves, J. Diff. Geometry 73, 1, 2006.

[15] L.C. Evans, Partial differential equations, Graduate Studies in Mathematics, 19, American Mathematical Society, Providence, RI, 1998.

[16] H. Hironaka, Subanalytic sets, Number theory, algebraic geometry and commutative algebra, in honor of Y. Akizuki, Tokyo, 1973.

[17] P.-L. Lions, Generalized solutions of Hamilton-Jacobi equations, Pitman, 1982. 
[18] W. S. Liu, H. J. Sussmann, Shortest paths for sub-Riemannian metrics of rank two distributions, Memoirs AMS 118, 564, 1995.

[19] R. Montgomery, Abnormal minimizers, SIAM J. Control Optim. 32, no. 6, 1994, 1605-1620.

[20] H.J. Pesch, A practical guide to the solution of real-life optimal control problems. Parametric optimization. Control Cybernet. 23, no. 1-2, 1994, 7-60.

[21] L. Pontryagin, V. Boltyanskii, R. Gamkrelidze, and E. Mischenko, The mathematical theory of optimal processes, Wiley Interscience, 1962.

[22] C. Prieur, E. Trélat, Quasi-optimal robust stabilization of control systems, to appear in SIAM J. Control Optim.

[23] L. Rifford, On the existence of local smooth repulsive stabilizing feedbacks in dimension three, to appear in J. Differential Equations.

[24] L. Rifford, The stabilization problem: AGAS and SRS feedbacks, in "Optimal Control, Stabilization, and Nonsmooth Analysis", Lectures Notes in Control and Information Sciences 301, Springer-Verlag, Heidelberg, 2004, 173-184.

[25] L. Rifford, E. Trélat, Morse-Sard type results in sub-Riemannian geometry, Math. Ann. 332, 1, 2005, 145-159.

[26] W. Rudin, Real and complex analysis, McGrow-Hill, 1966.

[27] A. Sarychev, The index of the second variation of a control system, Math. USSR Sbornik 41, $3,1982$.

[28] M. Tamm, Subanalytic sets in the calculus of variation, Acta Math. 146, 3-4, 1981, 167-199.

[29] E. Trélat, Some properties of the value function and its level sets for affine control systems with quadratic cost, J. Dyn. Cont. Syst. 6, 4, 2000.

[30] E. Trélat, Asymptotics of accessibility sets along an abnormal trajectory, ESAIM Control Optim. Calc. Var. 6, 2001.

[31] E. Trélat, Global subanalytic solutions of Hamilton-Jacobi type equations, Ann. Inst. Henri Poincaré, Analyse non Lin. 23, 3, 2006, 363-387.

[32] L. C. Young, Lectures on the calculus of variations and optimal control theory, Chelsea, New York, 1980. 